\title{
An Optimal Portfolio Problem of DC Pension with Input-Delay and Jump-Diffusion Process
}

\author{
Weixiang Xu $\mathbb{D}$ and Jinggui Gao \\ College of Mathematics and Systems Science, Shandong University of Science and Technology, Qingdao 266590, China \\ Correspondence should be addressed to Jinggui Gao; jingguigao@126.com
}

Received 5 June 2020; Accepted 10 July 2020; Published 31 July 2020

Guest Editor: Wenguang Yu

Copyright (C) 2020 Weixiang $\mathrm{Xu}$ and Jinggui Gao. This is an open access article distributed under the Creative Commons Attribution License, which permits unrestricted use, distribution, and reproduction in any medium, provided the original work is properly cited.

\begin{abstract}
In this paper, an optimal portfolio control problem of DC pension is studied where the time interval between the implementation of investment behavior and its effectiveness (hereafter input-delay) is particularly focused. There are two assets available for investment: a risk-free cash bond and a risky stock with a jump-diffusion process. And the wealth process of the pension fund is modeled as a stochastic delay differential equation. To secure a comfortable retirement life for pension members and also avoid excessive risk, the fund managers in this paper aim to minimize the expected value of quadratic deviations between the actual terminal fund scale and a preset terminal target. By applying the stochastic dynamic programming approach and the match method, the optimal portfolio control problem is solved and the closed-form solution is obtained. In addition, an algorithm is developed to calculate the numerical solution of the optimal strategy. Finally, we have performed a sensitivity analysis to explore how the managers' preset terminal target, the length of input-delay, and the jump intensity of risky assets affect the optimal investment strategy.
\end{abstract}

\section{Introduction}

In recent decades, the analysis and optimal control of pension investment strategy have become a hot topic as people have paid more attention on the security of retirement life. There are two major ways to manage pension funds [1]. One is the DB (defined-benefit) plans, whose pension is predetermined, and the financial risk is borne by the sponsor of the plan; the other is the DC (defined-contribution) schemes, whose contributions are preset and benefits determined by the returns on fund's portfolio. Compared to the DB pension plan, the advantage of DC plans is to transfer longevity risk and financial risk from the sponsor to the member. Due to the development of stock market and a fact that the population ageing is threatening the solvability of Pay-as-you-go public pension systems, the management of pensions is increasingly leaning towards DC plans rather than DB schemes $[2,3]$.

The stochastic optimal control theory has been extensively used to obtain the optimal investment strategy of DC pension. In [4], the stochastic optimal dynamic programming method was applied by Merton (1969) to solve the portfolio problem. In [5], with the salary risk and the inflation risk considered, the optimal investment proportion for DC pension in the stochastic interest framework was obtained by the help of the stochastic dynamic programming approach. In [6], an optimal asset allocation problem for DC pension was investigated where the price process of risky assets followed the Heston model, and the optimization problem was then solved via the stochastic dynamic programming method. It is worth noting that the price processes of risky assets have been described as a continuous process in the above literature.

In fact, emergencies such as Corona Virus Disease 2019, plunge in oil prices, policy intervention, and so on will cause that the price processes of the risky assets discontinuous and have jumps [7]. However, the sudden jumps in the price process of available assets could not be described by Brownian motion. To cope with the jumps, scholars gradually pay attention to the jump-diffusion model. In [8], an 
optimal portfolio policy and consumption problem was proposed under the environment where the price process of the stock was modeled as a log-normal jump-diffusion process. Their optimal portfolio fraction and optimal consumption were obtained with the help of the stochastic dynamic programming method. In [9], an optimal precommitment and equilibrium investment strategies problem was presented for DC pension, where the dynamics of the risky asset was modeled as a jump-diffusion process. The precommitment strategy was then found by the stochastic dynamic programming approach, while the equilibrium strategy was derived under the framework of game theory. In [10], Walter Mudzimbabwe investigated an optimal portfolio policy problem of DC pension under a special case of the market environment in [9], and he obtained the optimal numerical solution by applying the stochastic dynamic programming method and the bisection method. In [10], the impacts of the jump intensity and jump amplitude on the optimal portfolio policies were displayed. In [11], an optimal consumption and portfolio strategy problem was analyzed where the CPI, interest rate, index bonds and stocks were both modeled as jump-diffusion processes. And their optimal fraction of consumption and investment strategy was obtained by adopting the stochastic dynamic programming approach. Lately, in [12], taking into account that the reserve level of an insurance company can only be observed at discrete time points, periodic capital injection and barrier dividend strategy was considered in the compound Poisson risk model. The explicit expression of the Gerber-Shiu function in [12] was derived by means of the integral and differential method, and the expected discounted capital injection function and the expected discounted dividend function was derived on condition that the observation interval and claim amount are exponentially distributed.

The current international financial situation is changing rapidly, leading to the inevitable time interval from knowing the change in the economic situation to formulating the relevant investment strategies and, finally, the investment strategy taking effect [13]. To deal with the time interval, the optimal portfolio problem with delay was gradually focused by scholars. This can be found in [13-15]. In [14], an optimal portfolio and consumption problem was considered where the dynamics of the risky asset is modeled as a stochastic delay differential equation whose coefficients vary according to a stochastic factor. And their problem was solved by applying a dynamic programming approach. In [15], the wealth process of the insurer was modeled as a stochastic delay differential equation under which an optimal reinsurance and portfolio problem with jump-diffusion was investigated. The closed-form expression for the optimal strategy was then obtained by solving the derived HJB equation. For investors, with the time interval between the implementation of investment behavior and its effect (hereafter the input-delay) considered, the obtained optimal investment strategy will be more in line with the actual investment environment so that the investment risk can be reduced. Therefore, it is necessary to consider input-delay in solving the investment portfolio problem. In [13], the portfolio problem with input-delay was studied to maximize the expected return and the explicit expression of the optimal solution is obtained by applying the maximum principle. In terms of the optimal investment strategy for DC pension, affected by the input-delay, the duration that the investment behavior actually plays a role (hereafter acting duration) is shorter than the nominal management duration of DC pension (hereafter management duration). Thus, the fund manager of DC pension should formulate the optimal portfolio fraction with input-delay considered to make sure that the managers' preset terminal target could be achieved within the acting duration. However, one seldom studies the optimal asset allocation for DC pension with input-delay put into consideration.

In the optimal asset allocation problem of DC pension, scholars commonly aim to maximize the expected utility of terminal wealth $[1,5,16,17]$. Especially, to satisfy the basic requirement of fund members' retirement life, in [18], a minimum guarantee of the final wealth was calculated where the date of members' death was considered as a constant. Their objective was to maximize the expected utility of the final wealth exceeding the guarantee. A similar form of the objective in [18] can be found in $[3,19]$. In this circumstance, a large positive value of difference also means excessive risks, which may be unacceptable to some fund members. In [20], with the mortality considered, the managers aimed to minimize the accumulated deviations between a benefit outgo target and the actual benefit outgo during the whole distribution period and their optimal solution was obtained by applying the variational inequality method.

In this paper, we consider an optimal portfolio problem for DC pension with input-delay and jump-diffusion process. The financial market consists of a risk-free cash bond and a risky stock. During the nominal management duration of DC pension, the pension members are required to provide continuous contribution to managers. Fund managers invest pension wealth into available assets so that the pension fund scale is affected by the investment return. We assume that the pension fund is fully invested in cash bond after members' retirement (i.e., after the terminal time) and converted into annuities.

To obtain the numerical solution of the optimal investment strategies in the above situation, the main contributions of this paper are listed as follows:

(i) Put into consideration both the input-delay (i.e., the time interval between the implementation of investment behavior and its effectiveness) and the jump-diffusion process. The dynamics of DC pension fund is modeled as a stochastic delay differential equation (SDDE).

(ii) A target of the terminal fund scale (hereafter the preset terminal target) is calculated with the mortality and the inflation considered. It reflects the required fund scale at retirement time to maintain high standard retirement life for members. To secure a comfortable retirement life for fund members while avoiding excessive risk, the goal in our model is to minimize the expected value of quadratic deviation between the actual terminal fund and the preset terminal target. 
(iii) An optimization algorithm is given for calculating the numerical solution of the optimal strategy with input-delay. Meanwhile, sensitivity analysis is performed to show the impact of the preset terminal target, the length of input-delay, and jump intensity on the optimal investment strategies.

The remainder of this paper is organized as follows. In Section 2, we introduce the price processes of available assets and model the dynamics of the pension fund as a SDDE. In Section 3, the optimal asset allocation problem of DC pension with input-delay and jump-diffusion process is presented. In Section 4, the stochastic dynamic programming approach and the match method are applied to solve the optimization problem. An algorithm is developed to obtain the numerical solution of the optimal strategies. In Section 5, the numerical simulation and sensitivity analysis are provided.

\section{The Financial Market}

In this section, the financial market environment is introduced under which the optimization problem is proposed. We consider two financial assets available for investment: a risk-free cash bond and a single risky stock.

As in [10], the instantaneous risk-free rate of the cash bond is assumed to be a constant in this paper. Thus the price process of cash bond can be described by the following:

$$
\mathrm{d} M(t)=r M(t) \mathrm{d} t, \quad M(0)=M_{0},
$$

where $M(t)$ describes the price of risk-free cash bond at $t$ and $M(0)$ indicates the initial price of risk-free cash bond. $t \in[0, T]$ and $T$ is a given positive finite constant denoting the investment time horizon of DC pension plan. $r$ denotes the instantaneous risk-free interest rate.

In this paper, the jumping behavior of stock is focused and described as a compound Poisson process. Thus, the stochastic dynamics of stock can be expressed by a jumpdiffusion process:

$$
\mathrm{d} S(t)=S(t)\left[c \mathrm{~d} t+\sigma \mathrm{d} W(t)+\mathrm{d} \sum_{i=1}^{N(t)} V_{i}\right], \quad S(0)=S_{0}
$$

where $S(t)$ is the price of stock at time $t$ and $S(0)$ represents the initial price of stock. $c$ and $\sigma$ are the expected return and the volatility of the stock, respectively. In order to capture the features of the real market, we assume that $c>r$ [21]. As in [10], all processes and random variables are assumed to be defined on a filtered probability space $(\Omega, F, P) .\{W(t), t \geq 0\}$ denotes a standard Brownian motion. $\sum_{i=1}^{N(t)} V_{i}$ is a compound Poisson process to describe the jumping behavior of stock. $N(t)$ is a Poisson process with jump intensity $\lambda$. $V_{i}(i=1, \ldots, N(t))$ are i.i.d variables denoting the jump amplitude with mean $\mu_{v}=E\left(V_{i}\right)$ and variance $\sigma_{v}^{2}=\operatorname{Var}\left(V_{i}\right)$. Considering that the occurrence of Brownian motion and jump-diffusion process is independent, we assume that $W(t), N(t)$, and $V_{i}(i=1, \ldots, N(t))$ are mutually independent.
We consider the investment fraction in stock as the control variable. The short sale of the financial assets (the cash bond and stock) is both permitted, so the admissible range of the investment fraction in stock $(\Pi)$ is not restricted, i.e., $\Pi=(-\infty,+\infty)$ [20]. In this paper, the pension members are required to provide continuous contribution during the nominal management duration of DC pension.

Then, based on the dynamics of available assets, the stochastic dynamics of the pension fund can be written by the following stochastic differential equation (SDE):

$$
\begin{aligned}
\mathrm{d} X(t)= & {[X(t)-X(t) \pi(t)] \frac{\mathrm{d} M(t)}{M(t)}+X(t) \pi(t) \frac{\mathrm{d} S(t)}{S(t)} } \\
& +l(t) \mathrm{d} t, \quad X(0)=X_{0},
\end{aligned}
$$

where $t \in[0, T] . X(t)$ represents the fund scale of DC pension at time $t$ and $X(0)$ is the initial pension fund scale. $l(t)$ means the contribution rate at $t . \pi(t)$ is the investment fraction in stock at $t$, with $\pi(t) \in \Pi$.

From the investment behavior, the optimal asset allocation problem of DC pension can be regarded as a sort of optimal investment strategy problem. In optimal investment strategy problems, SDE with a similar composition to SDE (3) is commonly used to describe the wealth process, especially the dynamic of pension fund scale. This can be found in [19, 22-25]. However, considering the time interval between the implementation of investment behavior and its effect, the acting duration of investment behavior is shorter than the nominal management duration of DC pension. Therefore, in order to make sure that the preset terminal target could be achieved within the acting duration, the fund manager should formulate the optimal portfolio fraction with input-delay considered. Thus, we introduce the input-delay into SDE (3). The input-delay is denoted as $\tau$ which is an exogenous variable determined in advance. Then, the investment strategy that acts at a time $t$ is actually the investment strategy carried out at time $t-\tau$. Correspondingly, the actual amount invested in stock and cash bond at $t$ is $X(t-\tau) \pi(t-\tau)$ and $X(t)-X(t-\tau) \pi(t-\tau)$. Therefore, we have the following:

$$
X(t)=X_{0}+l(t) \mathrm{d} t, \quad t \in[0, \tau) .
$$

The dynamics of the pension fund in $[\tau, T]$ could be rewritten as a SDDE as follows:

$$
\begin{aligned}
\mathrm{d} X(t) & =[X(t) r+X(t-\tau) \pi(t-\tau)(c-r)+l(t)] \mathrm{d} t \\
& +X(t-\tau) \pi(t-\tau) \sigma \mathrm{d} W(t) \\
& +X(t-\tau) \pi(t-\tau) \mathrm{d} \sum_{i=1}^{N(t)} V_{i}, \quad t \in[\tau, T] .
\end{aligned}
$$

\section{The Optimization Problem}

3.1. The Preset Terminal Target. In DC plans, there is a principal-agent relationship between pension members and managers. In this paper, we assume that managers preset a target of the terminal fund scale which reflects the required wealth at retirement time to maintain high standard 
retirement life for members. Since the value of the preset terminal target directly affects the optimal proportion in different assets, its formulation has become a key part of the optimal portfolio control problem. In this paper, we present the calculation of the terminal target with both the mortality credit and the inflation considered.

$$
L_{0}=\int_{0}^{\infty} \mathrm{NP} \cdot e^{g s} e^{-r s}{ }_{s} p_{x_{0}+T} \mathrm{~d} s,
$$

where $L_{0}$ represents the preset terminal target of the pension fund. NP is an exogenous variable which means the requirement benefit outgo per unit of time to maintain a high standard retirement life at time $T . g$ denotes the inflation. ${ }_{s} p_{x_{0}+T}$ is an actuarial symbol which means the conditional probability that a person is alive at the age $x_{0}+T$ and still alive at $x_{0}+T+s$. The De Moivre Model in [26] is introduced in this paper to describe the force of mortality function and calculate ${ }_{s} p_{x_{0}+T}$.

$$
\mu(x)=\frac{1}{\omega-x},
$$

where $\mu(x)$ is the force of mortality function at the age of $x$. $\omega$ is the maximum age of an alive person.

Then, we have the following:

$$
\begin{aligned}
L_{0} & =\int_{0}^{\infty} \mathrm{NP} \cdot e^{g s} e^{-r s} p_{x_{0}+T} \mathrm{~d} s \\
& =\int_{0}^{\infty} \mathrm{NP} \cdot e^{(g-r) s} e^{-\int_{0}^{s} \mu\left(x_{0}+T+v\right) \mathrm{d} v} \mathrm{~d} s \\
& =\frac{\mathrm{NP}}{r-g}\left(1+\frac{1}{\omega-x_{0}-T}\right) .
\end{aligned}
$$

3.2. The Optimal Portfolio Control Problem (OPCP). The comfortable retirement life of fund members would lose guarantee when the value of the actual terminal fund is much lower than the preset terminal target. Conversely, if the value of the actual terminal fund scale is much higher than the target, the fund members will bear excessive risk. Therefore, the absolute deviation between the value of the actual terminal fund scale and the preset terminal target should not be too large. For the sake of simplicity, the goal of the optimal asset allocation problem in this paper is decided as minimizing the expected value of quadratic deviation between the actual terminal fund scale and the preset terminal target, i.e.,

$$
\min _{\pi(t-\tau) \in \Pi} \mathrm{E}\left\{\left[X(T)-L_{0}\right]^{2}\right\} .
$$

Due to the existence of input-delay, the duration for carrying out an investment strategy and the duration of its effect are $[0, T-\tau]$ and $[\tau, T]$, respectively. Then, we have $t \in[\tau, T]$ in (9).

Then, the optimal portfolio control problem for DC pension with input-delay and jump-diffusion process (OPCP) can be expressed by the following equation:

$$
(\mathrm{OPCP}): \begin{cases}\min _{\pi(t-\tau) \in \Pi}, & \mathrm{E}\left\{\left[X(T)-L_{0}\right]^{2}\right\}, \\ \text { s.t., } & (4),\end{cases}
$$

where $t \in[\tau, T]$.

\section{The Optimal Solution and Optimization Algorithm}

4.1. The Optimal Solution of OPCP. In this paper, we define the value function as follows:

$$
H(t, x)=\min _{\pi(t-\tau) \in \Pi} \mathrm{E}\left\{\left[X(T)-L_{0}\right]^{2} \mid X(t)=x\right\},
$$

where $H(t, x)$ is assumed to be at least twice continuously differentiable in $x$ and once in $t$.

In order to derive the HJB equation for (OPCP), based on [27], we decompose the $\operatorname{SDDE}$ (5) into continuous changes $d_{\text {(cont) }} X(t)$ and discontinuous changes $d_{\text {(jump) }} X(t)$. From SDDE (5), $d_{\text {(cont) }} X(t)$ and $d_{\text {(jump) }} X(t)$ can be expressed by the following:

$$
\begin{aligned}
\mathrm{d}_{\text {(cont) }} X(t)= & {[X(t) r+X(t-\tau) \pi(t-\tau)(c-r)+l(t)] \mathrm{d} t } \\
& +X(t-\tau) \pi(t-\tau) \sigma \mathrm{d} W(t),
\end{aligned}
$$

while

$$
\mathrm{d}_{(\text {jump })} X(t)=X(t-\tau) \pi(t-\tau) \mathrm{d} \sum_{i=1}^{N(t)} V_{i} .
$$

Thus, the dynamics of $H(t, x)$ can also be decomposed into continuous changes and jump changes as follows:

$$
\begin{gathered}
\mathrm{d}_{(\text {cont })} H(t, X(t)) \simeq H_{t}(t, X(t)) \mathrm{d} t \\
+H_{x}(t, X(t)) \mathrm{d}_{(\text {cont })} X(t)+\frac{1}{2} H_{x x}(t, X(t)) \\
\left(\mathrm{d}_{\text {(cont) }} X(t)\right)^{2}, \\
\mathrm{~d}_{\text {(jump) }} H(t, X(t))=\left[H\left(t, X(t)+X(t-\tau) \pi(t-\tau) V_{1}\right)\right. \\
-H(t, X(t))] \mathrm{d} N(t) .
\end{gathered}
$$

Then, we have the following:

$$
\begin{aligned}
\mathrm{d} H(t, X(t))= & \mathrm{d}_{(\text {cont })} H(t, X(t))+\mathrm{d}_{(\text {jump })} H(t, X(t)) \\
= & H_{t}(t, X(t)) \mathrm{d} t+H_{x}(t, X(t)) \mathrm{d}_{(\text {cont })} X(t) \\
& +\frac{1}{2} H_{x x}(t, X(t))\left(\mathrm{d}_{(\text {cont })} X(t)\right)^{2} \\
& +\left[H\left(t, X(t)+X(t-\tau) \pi(t-\tau) V_{1}\right)\right. \\
& -H(t, X(t))] \mathrm{d} N(t) .
\end{aligned}
$$


Therefore, the HJB equation for (OPCP) can be derived and expressed as follows:

$$
\begin{aligned}
0= & \min _{\pi(t-\tau) \in \Pi}\left\{H_{t}+H_{x}[X(t) r+X(t-\tau) \pi(t-\tau)(c-r)\right. \\
& +l(t)]+\frac{1}{2} H_{x x} X^{2}(t-\tau) \pi^{2}(t-\tau) \sigma^{2}+\lambda \mathrm{E}[H(t, X(t) \\
& \left.\left.\left.+X(t-\tau) \pi(t-\tau) V_{1}\right)-H(t, X(t))\right]\right\} .
\end{aligned}
$$

With the boundary condition of the value function

$$
H(T, x)=\left(x-L_{0}\right)^{2},
$$

we try a form of the value function

$$
H(t, x)=P(t) x^{2}+Q(t) x+R(t),
$$

where $P(T)=1, Q(T)=-2 L_{0}, R(T)=L_{0}^{2} . P(t)$, and $R(t)$ are both positive while $Q(t)$ is negative.

Then, we can derive that

$$
\begin{aligned}
H_{t} & =P_{t}(t) x^{2}+Q_{t}(t) x+R_{t}(t), \\
H_{x} & =2 P(t) x+Q(t), \\
H_{x x} & =2 P(t) .
\end{aligned}
$$

Combining (16), (18), and (19), the HJB equation can be rewritten as follows:

$$
\begin{aligned}
0= & \min _{\pi(t-\tau) \in \Pi}\left\{P_{t}(t) X^{2}(t)+Q_{t}(t) X(t)+2 P(t) X(t)\right. \\
& {[X(t) r+X(t-\tau) \pi(t-\tau)(c-r)+l(t)] } \\
& +R_{t}(t)+Q(t)[X(t) r+X(t-\tau) \pi(t-\tau)(c-r) \\
& +l(t)]+P(t) X^{2}(t-\tau) \pi^{2}(t-\tau) \sigma^{2} \\
& +2 \lambda P(t) X(t) X(t-\tau) \pi(t-\tau) V_{1} \\
& +\lambda Q(t) X(t-\tau) \pi(t-\tau) V_{1} \\
& \left.+\lambda P(t) X^{2}(t-\tau) \pi^{2}(t-\tau) V_{1}^{2}\right\} .
\end{aligned}
$$

By the match method, we transform (20) into the following form:

$$
\begin{array}{r}
\begin{array}{r}
0=\min _{\pi(t-\tau) \in \Pi}\left\{\left[X(t-\tau) \sqrt{\left(\sigma^{2}+\lambda V_{1}^{2}\right) P(t)} \pi(t-\tau)+\frac{\left(c-r+\lambda V_{1}\right)(2 X(t) P(t)+Q(t))}{2 \sqrt{\left(\sigma^{2}+\lambda V_{1}^{2}\right) P(t)}}\right]^{2}\right. \\
+P_{t}(t) X^{2}(t)+Q_{t} X(t)+R_{t}(t)+2 P(t) X(t) l(t)+Q(t) l(t)
\end{array} \\
\left.+2 P(t) r X^{2}(t)+Q(t) r X(t)-\frac{\left(c-r+\lambda V_{1}\right)^{2}(2 X(t) P(t)+Q(t))^{2}}{4\left(\sigma^{2}+\lambda V_{1}^{2}\right) P(t)}\right\} . \\
\left\{\begin{array}{l}
P_{t}(t)+P(t)(2 r+k)=0, \\
P(T)=1,
\end{array}\right.
\end{array}
$$
as follows:

$$
\pi^{*}(t-\tau)=-\frac{\left(c-r+\lambda V_{1}\right)(2 X(t) P(t)+Q(t))}{2\left(\sigma^{2}+\lambda V_{1}^{2}\right) P(t) X(t-\tau)}, \quad t \in[\tau, T] .
$$

Combining with (21) and (22), we obtain the following:

$$
\begin{aligned}
0= & P_{t}(t) X^{2}(t)+Q_{t} X(t)+R_{t}(t)+2 P(t) r X^{2}(t)+Q(t) r X(t) \\
& +2 P(t) X(t) l(t)+Q(t) l(t)-\frac{\left(c-r+\lambda V_{1}\right)^{2}}{4\left(\sigma^{2}+\lambda V_{1}^{2}\right)} \\
& \cdot\left[4 X^{2}(t) P(t)+\frac{Q^{2}(t)}{P(t)}+4 X(t) Q(t)\right] .
\end{aligned}
$$

Letting the coefficient of the quadratic factor be zero in (23), we get the following ordinary differential equation related to $P(t)$ : where $k=-\left(c-r+\lambda V_{1}\right)^{2} /\left(\sigma^{2}+\lambda V_{1}^{2}\right)$.

Letting the coefficient of the linear factor be zero in (23), the following ordinary differential equation related to $Q(t)$ is obtained:

$$
\left\{\begin{array}{l}
Q_{t}(t)+Q(t)(r+k)+2 P(t) l(t)=0, \\
Q(T)=-2 L_{0} .
\end{array}\right.
$$

Through the separating variables method and the constant variation method, we can easily obtain the solution of (24) and (25), i.e.,

$$
\begin{aligned}
& P(t)=e^{(2 r+k)(T-t)} \\
& Q(t)=-2\left[\int_{t}^{T} e^{(3 r+2 k)(T-t)} l(s) \mathrm{d} s+L_{0}\right] e^{-(r+k)(T-t)} .
\end{aligned}
$$

Since $P(t), Q(t)$ are enough to describe $\pi^{*}(t-\tau)$ in (22), we omit the calculations of $R(t)$ here. Substituting the 
obtained $P(t)$ and $Q(t)$ into (22), then we have the following:

$$
\begin{aligned}
\pi^{*}(t-\tau)= & \frac{c-r+\lambda V_{1}}{\sigma^{2}+\lambda V_{1}^{2}}\{-X(t) \\
& \left.+\left[\int_{t}^{T} e^{(3 r+2 k)(T-s)} l(s) \mathrm{d} s+L_{0}\right] e^{-(3 r+2 k)(T-t)}\right\} \frac{1}{X(t-\tau)},
\end{aligned}
$$

where $t \in[\tau, T]$.

Through the closed-form of the optimal investment strategy in (27), we learn that the manager will formulate the current optimal portfolio fraction $\left(\pi^{*}(t)\right)$ based on the current state $(X(t))$ and the effect generated by previous strategies (i.e., $X(t+\tau))$. It is obvious that our result is different from previous research results once the input-delay is not 0 .

4.2. An Optimization Algorithm for OPCP. To obtain the numerical solution of the optimal investment strategy and show its sample trajectory, in this paper, an algorithm (OPCPA) is developed, and the split-step algorithm (see [28] for details) is adopted to calculate the numerical solution of (27). The detailed design procedure of OPCPA is as follows:

Step 1: set the value of the parameters $\left(r, c, \sigma, \lambda, \mu_{v}, \sigma_{v}\right.$, $\left.T, X_{0}, l(t), \tau, g, x_{0}, \omega, N P\right)$ and give the distribution function of the jump amplitude $(V)$.

Step 2: let $t=0, h=0.01$.

Step 3: calculate $\pi^{*}(t)$ by (4), (5) and (27).

Step 4: substitute the obtained $\pi^{*}(t)$ into (5) and combine with the split-step algorithm to give a numerical approximation of $X(t+\tau+h)$. Let $t=t+h$.

Step 5: if $0 \leq t \leq \tau$, return to Step 3; else if $\tau<t<T-\tau$, substitute $X(t)$ and $X(t+\tau)$ into (27) to calculate $\pi^{*}(t)$ and return to Step 4; else if $t>T-\tau$, go to Step 6 .

Step 6: draw the sample trajectory of the optimal investment strategy where $t \in[0, T-\tau]$.

\section{Numerical Simulation}

In this section, a numerical simulation is performed to demonstrate the effectiveness of our model. Consider a DC pension with $T=20$ (years). The pension contribution rate is assumed to be constant $l(t)=0.15$. The other parameters are $X_{0}=1$ (ten thousand dollars), $r=0.02, c=0.065$, $\sigma=0.4, \quad \lambda=0.1, \quad \mu_{v}=0.1, \quad \sigma_{v}=0.4, \quad \tau=0.2, \quad g=0.015$, $x_{0}=35, \omega=110, \mathrm{NP}=0.03122$. As in [9], we assume that the jump amplitude of stock is simulated with log-normal distribution, i.e.,

$$
V_{i}=R_{i}-1, \quad \ln R_{i} \sim N\left(\mu, \theta^{2}\right), \quad i=1,2,3, \ldots,
$$

where $\mu=2 \ln \left(1+\mu_{v}\right)-1 / 2 \ln \left(1+2 \mu v+\sigma_{v}^{2}\right), \quad \theta^{2}=\ln (1+$ $\left.2 \mu_{v}+\sigma_{v}^{2}\right)-2 \ln \left(1+\mu_{v}\right)$.

From the expression of the optimal investment strategy in (27), in addition to the parameters of the market model, we know that the optimal investment strategy carried out at

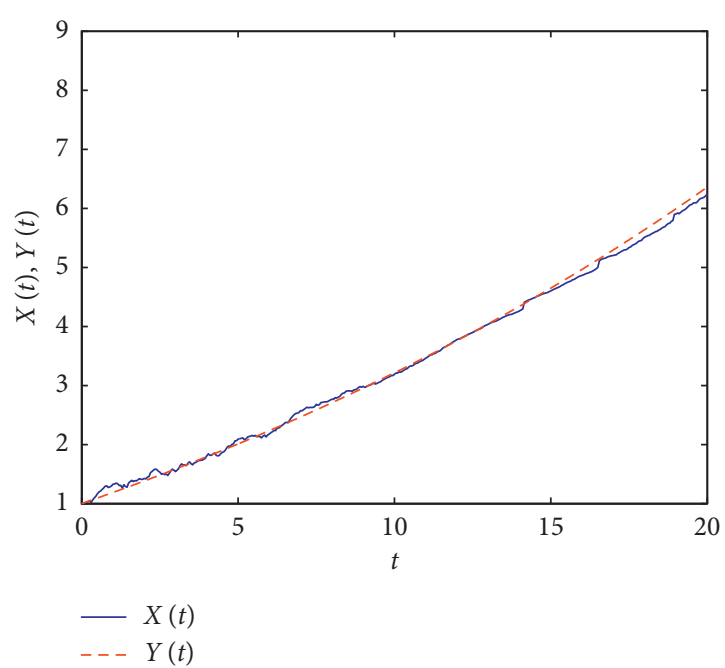

Figure 1: $X(t)$ is the pension fund scale at $t ; Y(t)$ represents the cumulative process of investing all of the pension fund in a risk-free asset with instantaneous interest rate $\delta . l(t)=0.15, r=0.02$, $c=0.065, \sigma=0.4, \lambda=0.1, \mu_{v}=0.1, \sigma_{v}=0.4, \tau=0.2, g=0.015$, $x_{0}=35, \omega=110, \mathrm{NP}=0.03122$.

time $t$ is also dependent on the pension fund at $t$ and the pension fund at $t+\tau$. We adopt the OPCPA algorithm to simulate the evolution process of the pension fund and the trajectory of the optimal investment strategy.

With step length $h=0.05$, we perform numerical simulation in two parts: simulating the trajectory of optimal investment strategy and the accumulation process of DC pension fund; sensitivity analysis including the impact of managers' preset terminal target, the length of input-delay, and jump intensity on the optimal investment strategies.

In order to facilitate the observation of the evolution process of the actual fund scale, we assume that the preset terminal target is the value of a risk-free asset at time $T$. The value of the risk-free asset at time $t$ can be expressed as $Y(t)=X_{0} \cdot e^{\delta t}+\int_{0}^{t} l(s) e^{\delta(t-s)} \mathrm{d} s, \quad Y(T)=L_{0} \quad$ and $Y(0)=X_{0}$. The dynamics of $Y(t)$ describes the cumulative process of investing all of the pension fund in a risk-free asset with an instantaneous interest rate $\delta$. Combining with (8), we have $\delta=0.035$. In the numerical simulation, we will compare the evolution process of $Y(t)$ with $X(t)$.

Figure 1 shows the evolution process of pension fund under the obtained optimal investment strategy in our model. Due to the input-delay, the duration of carrying out investment strategy is $[0, T-\tau]$ while the duration of its effect is $[\tau, T]$. The results show a relatively larger increase of fund scale in the first two years and a relatively gentle increase in the later stage. From Figure 1, we know that the optimal investment strategy obtained via our model can satisfy the manager's goal; that is, the DC pension fund scale is close to the managers' preset terminal target at the terminal moment.

Figure 2 shows the trajectory of the optimal investment strategy obtained in our model. The duration of carrying out the optimal investment strategies is $[0, T-\tau]$. It is clear that $\pi^{*}(t)$ presents a wave-like decline over time. The relatively high proportion investing in stock in the initial stage has 


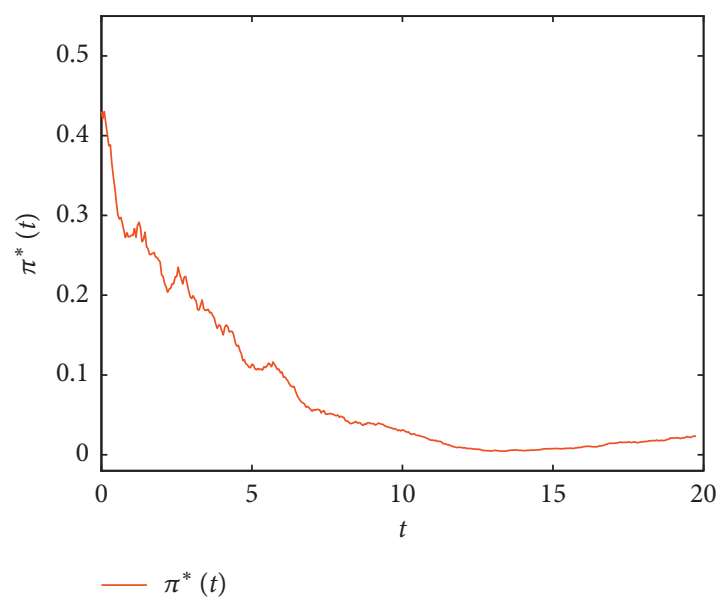

FIgURE 2: The optimal investment fraction in stock obtained in our model. $l(t)=0.15, r=0.02, c=0.065, \sigma=0.4, \lambda=0.1, \mu_{v}=0.1$, $\sigma_{v}=0.4, \tau=0.2, g=0.015, x_{0}=35, \omega=110, \mathrm{NP}=0.03122$.

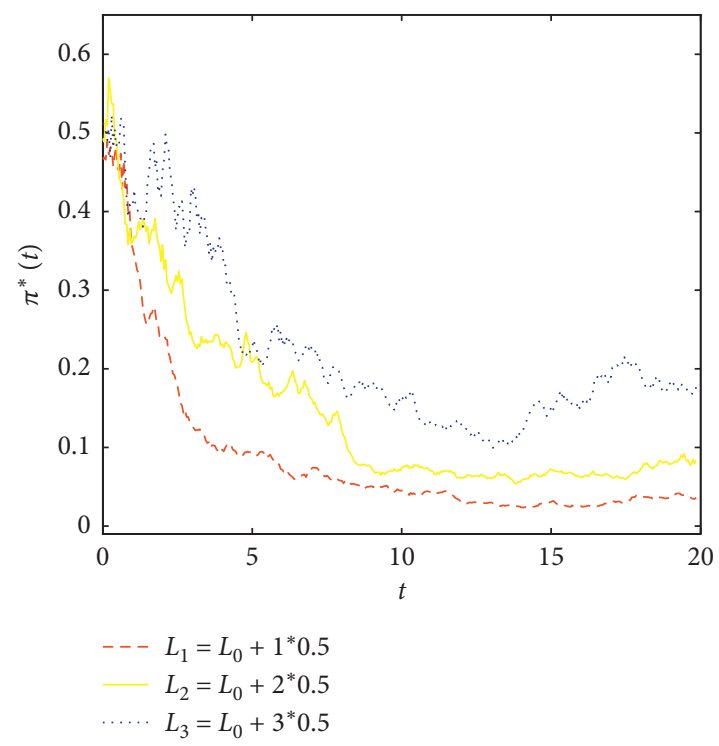

FIgURE 3: The impact of managers' preset terminal target on the optimal investment strategy $\pi^{*}(t) . l(t)=0.15, r=0.02, c=0.065$, $\sigma=0.4, \lambda=0.1, \mu_{v}=0.1, \sigma_{v}=0.4, \tau=0.1, g=0.015, x_{0}=35$, $\omega=110, N P=0.03122 . L_{i}(i=1,2,3)$ represents the level of the different preset terminal target.

resulted in a significant increase of pension fund, which has led to less demand for stock in the later stages to avoid unnecessary risks.

Figure 3 shows the impact of the managers' preset terminal target on the optimal investment fraction in stock. The results show that increasing the preset terminal target $\left(L_{0}\right)$ increases the optimal investment fraction in stock. This result is in line with reality. The higher the preset terminal target, the more the stock needed to significantly increase the pension fund scale and meet the preset terminal target.

Figure 4 shows the effect of the length of input-delay on the optimal investment fraction in stock. The results show that the longer the length of input-delay, the higher the

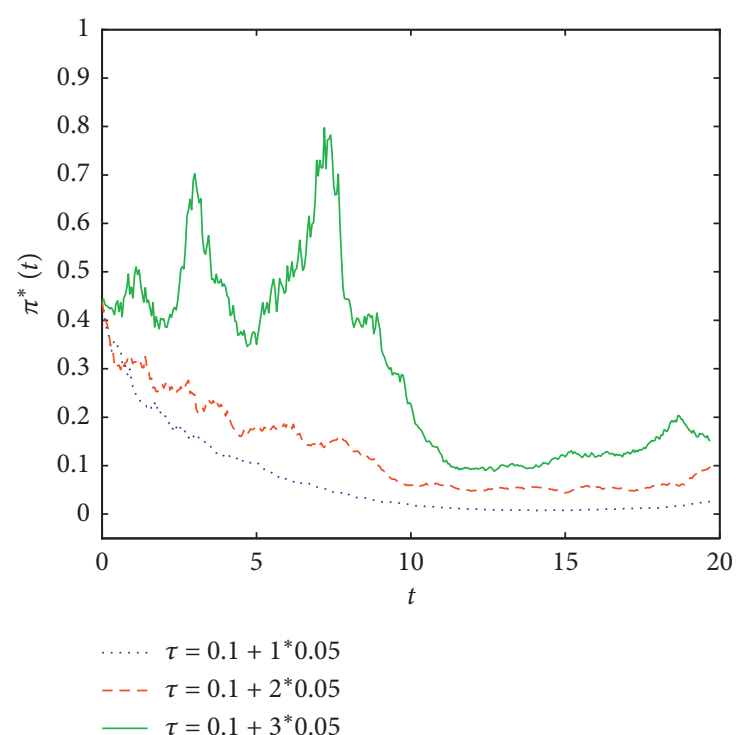

FIgURE 4: The impact of the length of input-delay $\tau$ on optimal investment strategy $\pi^{*}(t) . l(t)=0.15, r=0.02, c=0.065, \sigma=0.4$, $\lambda=0.1, \quad \mu_{v}=0.1, \quad \sigma_{v}=0.4, \quad g=0.015, \quad x_{0}=35, \quad \omega=110$, $\mathrm{NP}=0.03122$.

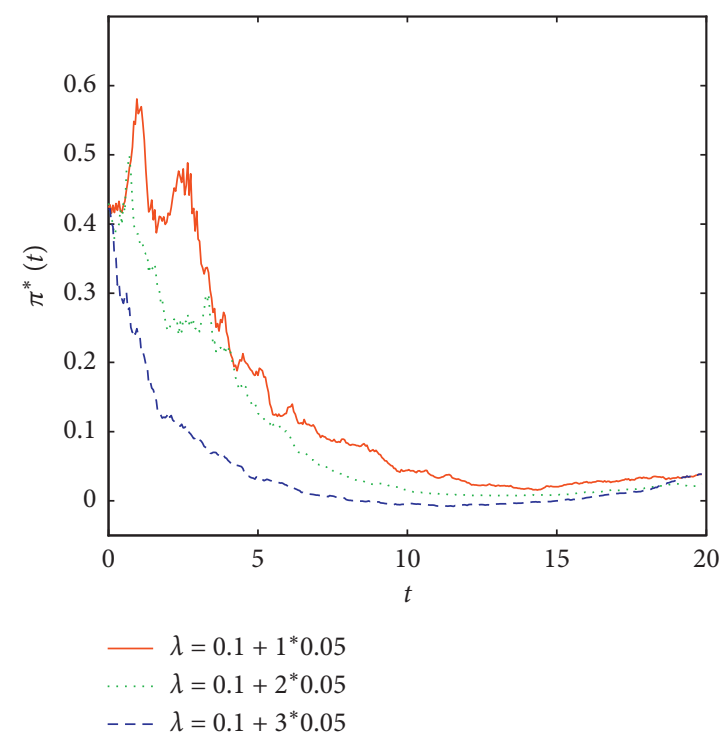

FIgURE 5: The impact of the jump intensity $\lambda$ on an optimal investment strategy. $l(t)=0.15, r=0.02, c=0.065, \sigma=0.4, \mu_{v}=0.1$, $\sigma_{v}=0.4, \tau=0.1, g=0.015, x_{0}=35, \omega=110, \mathrm{NP}=0.03122$.

optimal investment proportion in stock. Due to the existence of input-delay, the acting duration of investment behavior is $[\tau, T]$. Increasing the length of input-delay reduces the length of acting duration. In this situation, the investment proportion of stock should be increased to meet the managers' preset terminal target at the terminal time.

Figure 5 shows the influence of the jump intensity of stock on optimal investment proportion in stock. From Figure 5, we know that the increasing jump intensity decreases the optimal investment proportion in stock. Large $\lambda$ implies great uncertainty of financial market and also unknown risk that fund members have to bear, that is, the great 
investment risk. Therefore, in this circumstance, the proportion invested in stock should be reduced to avoid unknown great risk.

\section{Conclusion}

In this paper, we studied an optimal DC pension plan, where the input-delay is considered and the price process of the risky asset is modeled as a jump-diffusion process. Due to the input-delay, the accumulation process of DC pension fund is expressed as a SDDE. Considering the principalagent relationship between pension members and managers, we present the calculation of a terminal target with both the mortality credit and the inflation included, which reflects the required fund scale at the terminal time to provide high standard retirement life for members. To secure a comfortable retirement life for pension members and avoid excessive risk, the management goal of our model is to minimize the expected value of the quadratic deviations between the actual terminal fund and the preset terminal target. In order to solve the optimal portfolio control problem, the stochastic dynamic programming method is adopted. Then, the closed-form solution of the resulting $\mathrm{HJB}$ equation is obtained by applying the match method. In addition, an algorithm is given in this paper to calculate the numerical solution of the optimal investment strategy. With the help of the optimization algorithm, we simulated the trajectory of the optimal investment strategy and the accumulation process of the DC pension under the optimal strategy. The results show that our model could satisfy the management goal. And we also conducted a sensitivity analysis to explore the impact of the preset terminal target, the length of the input-delay and the jump intensity of stock on the optimal investment strategy.

Our findings show that the increasing preset terminal target increases the optimal investment proportion of stock to satisfy the management goal. The longer the length of input-delay, the shorter the acting duration of investment behavior, the larger the optimal investment fraction of stock to improve the performance of pension fund in a shorter duration and managers should decrease the proportion of investing in stock when jump intensity of stock is high to avoid unknown great risk.

\section{Data Availability}

No data were used to support this study.

\section{Conflicts of Interest}

The authors declare that they have no conflicts of interest.

\section{Acknowledgments}

This work was supported by the Natural Science Foundation of Shandong Province, China (Grant no. ZR2015FM014), Postdoctoral Science Foundation of China (Grant no. 2015M572060), and Postdoctoral Science Foundation of Qingdao, China.

\section{References}

[1] P. Battocchio and F. Menoncin, "Optimal pension management in a stochastic framework," Insurance: Mathematics and Economics, vol. 34, no. 1, pp. 79-95, 2004.

[2] G. Guan and Z. Liang, "Optimal management of DC pension plan under loss aversion and value-at-risk constraints," Insurance: Mathematics and Economics, vol. 69, pp. 224-237, 2016.

[3] Y. Dong and H. Zheng, "Optimal investment of DC pension plan under short-selling constraints and portfolio insurance," Insurance: Mathematics and Economics, vol. 85, pp. 47-59, 2019.

[4] R. C. Merton, "Lifetime portfolio selection under uncertainty: the continuous-time case," The Review of Economics and Statistics, vol. 51, no. 3, pp. 247-257, 1969.

[5] P. Battocchio and F. Menoncin, "Optimal portfolio strategies with stochastic wage income: the case of a defined contribution pension plan," Discussion Papers (IRES-Institut de Recherches Economiques et Sociales), Noisy-le-Grand, France, 2002.

[6] J. Ma, H. Zhao, and X. Rong, "Optimal investment strategy for a DC pension plan with mispricing under the Heston model," Communications in Statistics-Theory and Methods, vol. 49, no. 13, pp. 3168-3183, 2020.

[7] M. Li, Y. Deng, Y. Huang, and H. Ou, "Optimal strategies for an ambiguity-averse insurer under a jump-diffusion model and defaultable risk," Mathematical Problems in Engineering, vol. 2020, Article ID 6207805, 20 pages, 2020.

[8] F. B. Hanson and J. J. Westman, "Optimal consumption and portfolio control for jump-diffusion stock process with lognormal jumps," in Proceedings of the 2002 American Control Conference (IEEE Cat. No. CH37301), Anchorage, AK, USA, May 2002.

[9] J. Sun, Z. Li, and Y. Zeng, "Precommitment and equilibrium investment strategies for defined contribution pension plans under a jump-diffusion model," Insurance: Mathematics and Economics, vol. 67, pp. 158-172, 2016.

[10] W. Mudzimbabwe, "A simple numerical solution for an optimal investment strategy for a DC pension plan in a jump diffusion model," Journal of Computational and Applied Mathematics, vol. 360, pp. 55-61, 2019.

[11] C. I. Nkeki, "Optimal pension fund management in a jumpdiffusion environment: theoretical and empirical studies," Journal of Computational and Applied Mathematics, vol. 330, pp. 228-252, 2018.

[12] W. Yu, P. Guo, Q. Wang et al., "On a periodic capital injection and barrier dividend strategy in the compound Poisson risk model," Mathematics, vol. 8, no. 4, p. 511, 2020.

[13] L. Li, Study on Mean-Variance Portfolio Selection with Time Delay, Shandong University, Jinan, China, 2016.

[14] L. Li and H. Mi, "Optimal investment and consumption with stochastic factor and delay," The ANZIAM Journal, vol. 61, no. 1, pp. 99-117, 2019.

[15] Q. Zhang and P. Chen, "Optimal reinsurance and investment strategy for an insurer in a model with delay and jumps," Methodology and Computing in Applied Probability, vol. 22, no. 2, pp. 777-801, 2020.

[16] J. Gao, "Stochastic optimal control of DC pension funds," Insurance: Mathematics and Economics, vol. 42, no. 3, pp. 1159-1164, 2008.

[17] H. Chang, C. Wang, Z. Fang, and D. Ma, "Defined contribution pension planning with a stochastic interest rate and mean-reverting returns under the hyperbolic absolute risk 
aversion preference," IMA Journal of Management Mathematics, vol. 31, no. 2, pp. 167-189, 2020.

[18] J.-F. Boulier, S. Huang, and G. Taillard, "Optimal management under stochastic interest rates: the case of a protected defined contribution pension fund," Insurance: Mathematics and Economics, vol. 28, no. 2, pp. 173-189, 2001.

[19] G. Guan and Z. Liang, "Optimal management of DC pension plan in a stochastic interest rate and stochastic volatility framework," Insurance: Mathematics and Economics, vol. 57, pp. 58-66, 2014.

[20] L. He and Z. Liang, "Optimal assets allocation and benefit outgo policies of DC pension plan with compulsory conversion claims," Insurance: Mathematics and Economics, vol. 61, pp. 227-234, 2015.

[21] D. Li, X. Rong, H. Zhao, and B. Yi, "Equilibrium investment strategy for DC pension plan with default risk and return of premiums clauses under CEV model," Insurance: Mathematics and Economics, vol. 72, pp. 6-20, 2017.

[22] J. Gao, "Optimal portfolios for DC pension plans under a CEV model," Insurance: Mathematics and Economics, vol. 44, no. 3 , pp. 479-490, 2009.

[23] Q.-P. Ma, "On "optimal pension management in a stochastic framework" with exponential utility," Insurance: Mathematics and Economics, vol. 49, no. 1, pp. 61-69, 2011.

[24] P. Wang and Z. Li, "Robust optimal investment strategy for an AAM of DC pension plans with stochastic interest rate and stochastic volatility," Insurance: Mathematics and Economics, vol. 80, pp. 67-83, 2018.

[25] X. Zhang and X. Zheng, "Optimal investment-reinsurance policy with stochastic interest and inflation rates," Mathematical Problems in Engineering, vol. 2019, Article ID 5176172, 14 pages, 2019.

[26] H.-P. Kohler and I. V. Kohler, "Frailty modeling for adult and old age mortality: the application of a modified de moivre hazard function to sex differentials in mortality," Demographic Research, vol. 3, no. 1, 2000.

[27] F. Hanson, Applied Stochastic Processes and Control for JumpDiffusions: Modeling, Analysis, and Computation, Society for Industrial and Applied Mathematics, Philadelphia, PA, USA, 2007.

[28] Y. Qiu, Z. Wang, and Y. Qin, "Split-step algorithm for nonlinear stochastic delay differential equations with jumps," Journal of East China University of Science and Technology (Natural Science Edition), vol. 40, p. 816, 2014. 